\title{
Infrared spectroscopy of electronic bands in bilayer graphene
}

\author{
A. B. Kuzmenko, E. van Heumen, and D. van der Marel \\ Départment de Physique de la Matière Condensée, Université de Genève, CH-1211 Genève 4, Switzerland \\ P. Lerch \\ Paul Scherrer Institute, Villigen 5232, Switzerland \\ P. Blake, K. S. Novoselov, and A. K. Geim \\ Manchester Centre for Mesoscience and Nanotechnology, University of Manchester, Manchester M13 9PL, United Kingdom
}

(Received 14 October 2008; published 30 March 2009)

\begin{abstract}
We present infrared spectra $(0.1-1 \mathrm{eV})$ of electrostatically gated bilayer graphene as a function of doping and compare it with tight-binding calculations. All major spectral features corresponding to the expected interband transitions are identified in the spectra: a strong peak due to transitions between parallel split-off bands and two onset-like features due to transitions between valence and conduction bands. A strong gate voltage dependence of these structures and a significant electron-hole asymmetry are observed that we use to extract several band parameters. The structures related to the gate-induced band gap are less pronounced in the experiment than predicted by the tight-binding model that uses parameters obtained from previous experiments on graphite and recent self-consistent band-gap calculations.
\end{abstract}

DOI: 10.1103/PhysRevB.79.115441

PACS number(s): 78.30.Na, 78.20.-e, 78.67.Pt, 81.05.Bx

\section{INTRODUCTION}

Since the first successful attempt to isolate graphene, ${ }^{1}$ this two-dimensional material remains in the focus of active research motivated by a unique combination of electronic properties and a promising potential for applications. ${ }^{2}$ Its infrared response, like many other transport and spectral properties, is notably distinct from the one of conventional metals and semiconductors. For example, the optical conductance $\operatorname{Re} G(\omega)$ of monolayer graphene, which describes the photon absorption by a continuum of electronic transitions between the hole and electron conical bands, remains constant in a broad range of photon energies and equal to $G_{0}=e^{2} / 4 \hbar .^{3-5}$ Quite remarkably, the optical transmittance of single carbon layer in this range depends solely on the fine-structure constant. ${ }^{4,6}$ In bilayer graphene, where the interlayer electron hopping results in two extra electron and hole bands separated from the main bands by about $0.4 \mathrm{eV}$, one expects to see a set of intense and strongly doping-dependent infrared structures $^{7-9}$ sensitive to various band details and quasiparticle scattering rates. This makes infrared spectroscopy a powerful probe of the low-energy electronic dispersion in graphene, especially in combination with a possibility to electrostatically control the doping level. ${ }^{5,10,11}$ Here we present infrared spectra of bilayer graphene crystals in a broad doping range, which allows us to observe several important features, in particular a significant electron-hole asymmetry. By comparing data with the tight-binding Slonczewski-Weiss-McClure (SWMcC) model, ${ }^{12}$ we identify interband transitions and determine some band parameters.

Bilayer graphene is considered to be particularly important for electronics applications by virtue of a band gap that opens when a difference between the electrostatic potential of the two layers is introduced, either by chemical doping or by applying gate voltage. ${ }^{13-18}$ Angle-resolved photoemission (ARPES) measurements indicate such a gap in potassiumdoped bilayer graphene epitaxially grown on SiC. ${ }^{16}$ Although transport experiments ${ }^{17,18}$ demonstrate that a band gap also opens in gate-tunable bilayer graphene flakes, no spectroscopic information about the size of the gate-induced gap is currently available. The analysis of infrared data opens a unique opportunity to address this issue quantitatively.

\section{EXPERIMENT}

The sample used in this study is a large $(\sim 100 \mu \mathrm{m})$ bilayer graphene flake (Graphene Industries Ltd.) on top of an $n$-doped $\mathrm{Si}$ substrate covered with a $300 \mathrm{~nm}$ layer of $\mathrm{SiO}_{2}$ [Fig. 1(a)]. A field-effect device configuration allowed us to simultaneously measure the dc resistivity and infrared reflectance as functions of the applied gate voltage $V_{g}$. Optical spectra in the photon energy range of $0.1-1 \mathrm{eV}$ were collected at the temperature of the substrate $\approx 10 \mathrm{~K}$ with an infrared microscope (Bruker Hyperion 2000) focusing the beam on a spot of about $30 \mu \mathrm{m}$ in diameter. The absolute reflectance of graphene, $R_{\text {flake, }}$ and of the bare substrate, $R_{\text {oxide }}$, [Fig. 1(b)] were obtained by using a circle of gold deposited close to the sample as a reference mirror. The bare substrate spectrum features intense optical phonon modes in $\mathrm{SiO}_{2}$ below $0.15 \mathrm{eV}$ and a dip at $0.7 \mathrm{eV}$ due to the FabryPerot effect in the $\mathrm{SiO}_{2}$ layer. The change in the absolute reflectivity introduced by graphene $\Delta R=R_{\text {flake }}-R_{\text {oxide }}$ is small but reproducibly measurable as we checked on a second sample. By taking difference spectra, we largely cancel spurious optical effects such as a weak $0.4 \mathrm{eV}$ absorption band due to some frozen water. The resistivity maximum that corresponds to zero doping [Fig. 1(b), inset] is found to be at $V_{g 0}=-25 \mathrm{~V}$ instead of $0 \mathrm{~V}$, which we attribute to a charging effect by contaminant molecules.

\section{OPTICAL SPECTRA}

The curves of $\Delta R(\omega)$ between 0.2 and $0.6 \mathrm{eV}$ are shown in Fig. 2(a) as a function of the gate voltage from -100 to 

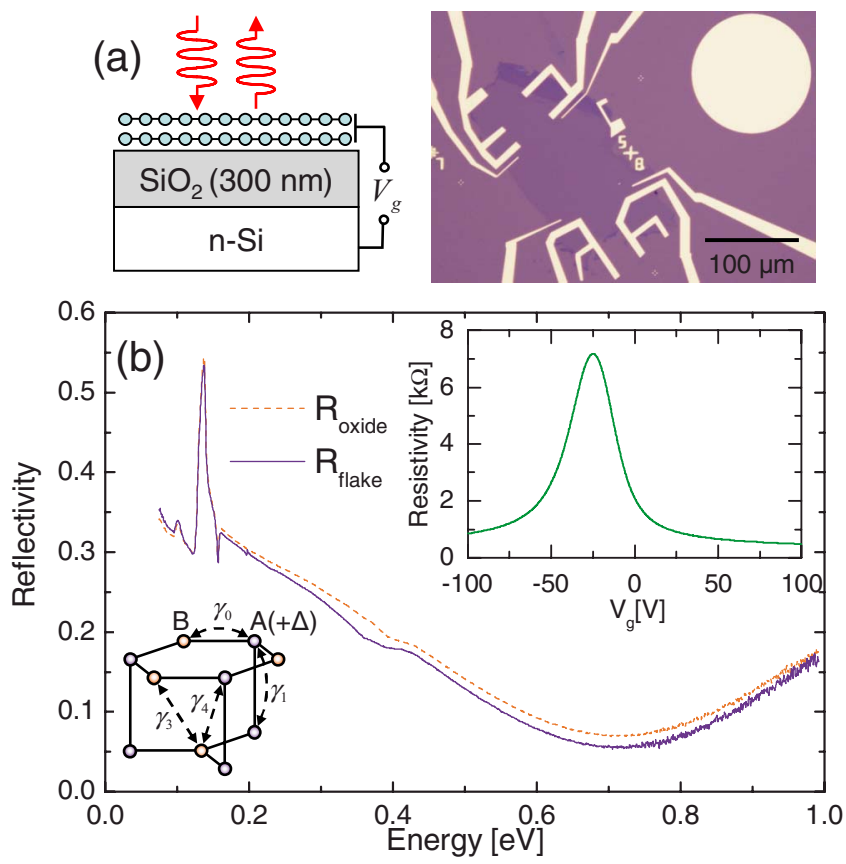

FIG. 1. (Color online) (a) Schematic view and a micrograph of the used bilayer graphene device. The flake is seen as a darker area between the contacts. (b) Infrared reflectance of graphene flake (blue solid line) and of bare substrate (red dotted line) (taken at $T$ $=10 \mathrm{~K}$ and $V_{g}=+100 \mathrm{~V}$ ). Left inset: Bernal stacking of bilayer graphene and relevant hopping terms. Right inset: resistivity at 10 $\mathrm{K}$ as a function of the gate voltage.

$+100 \mathrm{~V}$. The spectra in this region are very sensitive to the gate voltage and show a significant asymmetry between the electron $\left(V_{g}>V_{g 0}\right)$ and the hole $\left(V_{g}<V_{g 0}\right)$ dopings. Since the measured reflectivity depends on both real and imaginary parts of the complex dielectric function $\epsilon(\omega)$ as well as on the substrate optical properties, it is more convenient to discuss the data in terms of the real part of the optical bilayer conductance $G(\omega)$, which is related to the optical conductivity $\sigma(\omega)=\epsilon(\omega) \omega /(4 \pi i)$ by the relation $G(\omega)=\sigma(\omega) d$, where $d=6.7 \AA$ is the double interlayer distance. We extracted this quantity by a Kramers-Kronig (KK) constrained inversion ${ }^{19}$ of the raw reflectivity data. Due to a sensitivity of the inversion procedure to the systematic uncertainty $(\sim 0.005)$ of $\Delta R$ and to the data extrapolations beyond the experimental spectral range (we used graphite optical data ${ }^{6}$ as the most reasonable extrapolation) the inverted function $\operatorname{Re} \widetilde{G}(\omega)$ is likely to contain a spectrally smooth background as compared to Re $G(\omega)$. Although this background does not allow us to determine accurately the absolute conductance, it affects the positions of spectral structures and their doping dependence to a much lesser extent.

The spectra of $\operatorname{Re} \widetilde{G}(\omega)$ [Fig. 2(b)] reveal a prominent peak centered between 0.35 and $0.4 \mathrm{eV}$, whose intensity increases with the absolute value of the gate voltage and vanishes as $V_{g}$ approaches $V_{g 0}$. Based on previous theoretical works $^{7-9}$ as well as on the calculations described below we assign this peak to a transition between the hole bands 1 and 2 [marked as $\mathrm{C}$ in Fig. 3(e)] for $V_{g}<V_{g 0}$ and to the one between the electron bands 3 and 4 (marked as B) for $V_{g}$
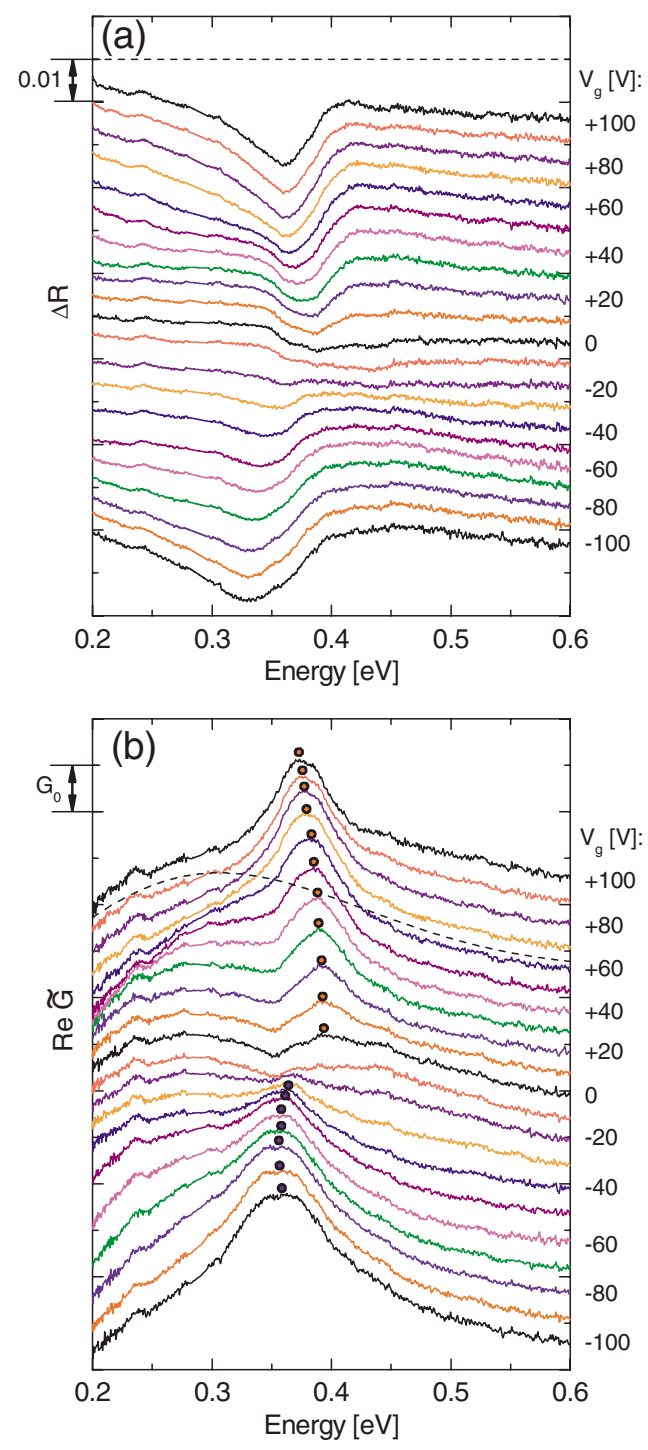

FIG. 2. (Color online) (a) Midinfrared spectra of $\Delta R$ at $T$ $\approx 10 \mathrm{~K}$ as a function of the gate voltage $V_{g}$. The curves are separated by 0.005 ; the dashed line is the zero level for the $+100 \mathrm{~V}$ curve. (b) Real part of the infrared sheet conductance of bilayer graphene $\tilde{G}(\omega)$, derived from the reflectance curves [panel (a)] using a Kramers-Kronig inversion. The curves are separated by $0.5 G_{0}$. Note that $\widetilde{G}(\omega)$ possibly differs from the true conductance $G(\omega)$ by a spectrally featureless gate-independent background, as explained in the text. The dashed line is the correction (shown relative to the $+100 \mathrm{~V}$ spectrum) used to generate Fig. 3(b).

$>V_{g 0}$. The doping-induced shift of the Fermi level away from the Dirac point expands the momentum space, where this transition is allowed by the electronic occupation of the initial and the final states, and therefore increases the infrared intensity of the peak.

The energy of this peak is given by the band separation and is close to the interlayer vertical hopping parameter $\gamma_{1}$ [shown in the inset of Fig. 1(b)]. In the case of precisely symmetric electron and hole bands, one would expect the same peak position for the positive and negative gate voltages. However, the data reveal a clear asymmetry: at positive voltages the maximum [marked with red circles in Fig. 2(b)] 

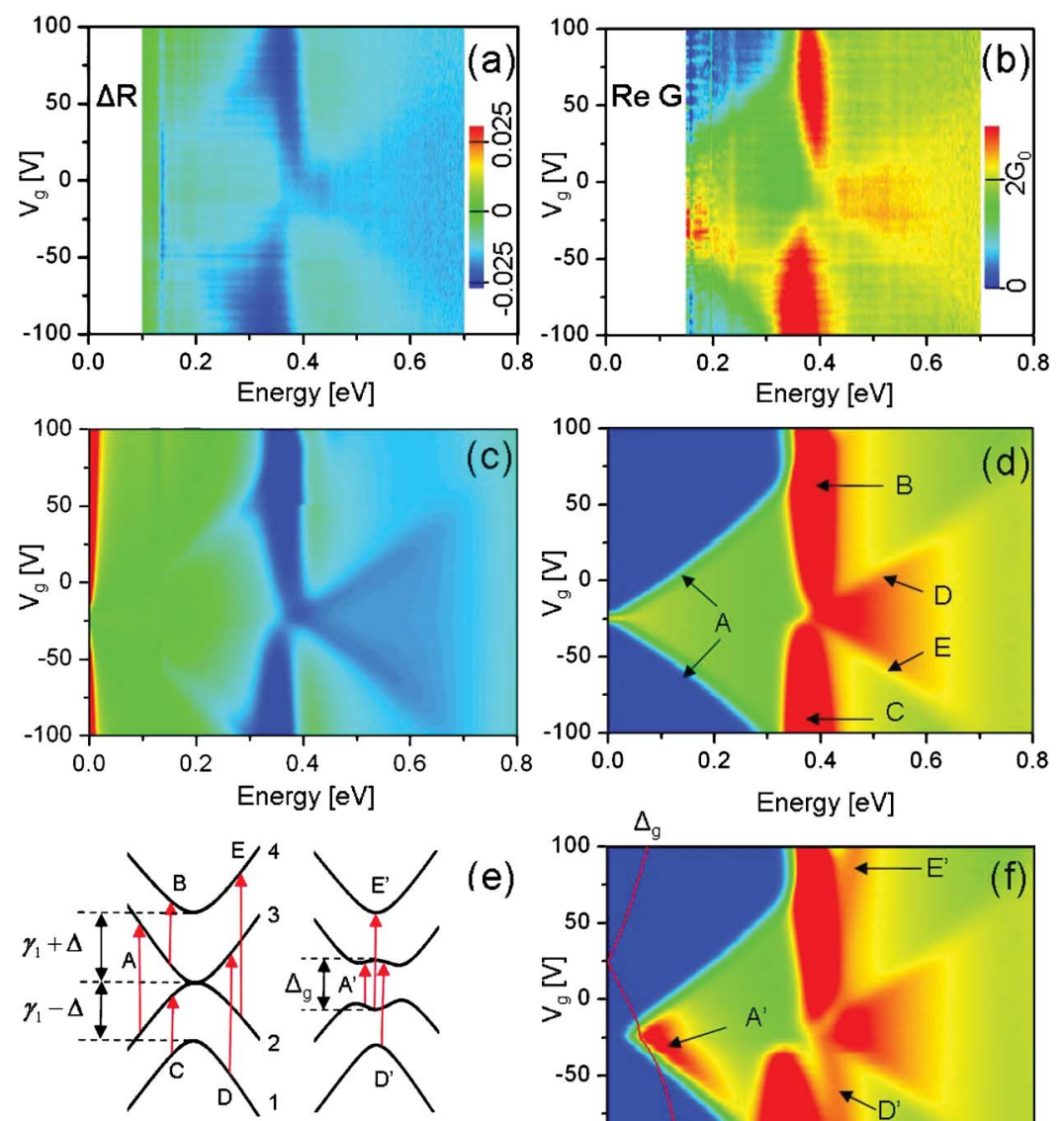

(e)

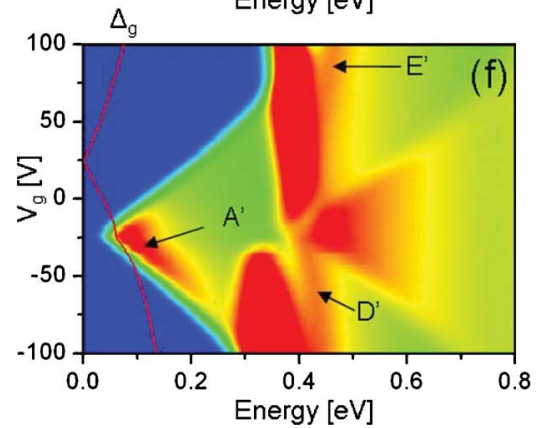

FIG. 3. (Color online) (a) and (b) Color plots of the raw $\Delta R(\omega)$ and the derived $\operatorname{Re} G(\omega)$ spectra as a function of $\omega$ and $V_{g}$. (c) and (d) $\Delta R$ and $\operatorname{Re} G(\omega)$ calculated using the tight-binding model assuming that the band gap is zero. (e) The four bands of bilayer graphene in the absence (left) and in the presence (right) of the band gap, with the interband transitions shown with arrows. (f) $\operatorname{Re} G(\omega)$ calculated assuming that the band gap $\Delta_{g}$ is present as given by the red solid curve (Ref. 17). is higher in energy and shows a much stronger dependence on $V_{g}$ than at negative voltages (blue circles). As was pointed out in Refs. 20 and 21, the energy of the peak on the electron and hole side taken close to the charge neutral point $\left(V_{g 0}\right.$ $=-25 \mathrm{~V}$ in our case) is equal to $\gamma_{1}+\Delta$ and $\gamma_{1}-\Delta$, respectively, where the parameter $\Delta$ is the potential difference between carbon sites $\mathrm{A}$ and $\mathrm{B}$. These values in our case are $0.393 \pm 0.005 \mathrm{eV}$ and $0.363 \pm 0.005$, which yields $\gamma_{1}$ $=0.378 \pm 0.005 \mathrm{eV}$ and $\Delta=0.015 \pm 0.005 \mathrm{eV}$. The value of $\gamma_{1}$ is very close to $0.377 \mathrm{eV}$ found in graphite. ${ }^{22}$ However, it is somewhat smaller than $0.404 \mathrm{eV}$ reported in Refs. 20 and 21 for bilayer graphene flake. This suggests that the interlayer distance, to which $\gamma_{1}$ is the most sensitive, may change from sample to sample. As far as $\Delta$ is concerned, there is much less agreement on the value of this parameter in graphite in the literature. While the magnetoreflection and de Haas-van Alphen measurements suggest that $\Delta$ is $-0.008 \mathrm{eV}$ (see Ref. 22, and references therein), infrared data ${ }^{6,23}$ give a value of $+0.04 \mathrm{eV}$. Our value agrees in sign with the infrared-based estimate in graphite but is about 2-3 times smaller. This difference can be understood using electrostatics arguments. In Bernal stacked graphite, each carbon layer is symmetrically surrounded by two other layers, in contrast to bilayer graphene. Therefore one may expect the difference between the (screened) Coulomb potential on sites A and B induced by charges on other layers to be larger in graphite.

\section{COMPARISON TO THE TIGHT-BINDING MODEL}

In order to get further insight, we compare the experimental data with calculations based on the tight-binding SWMcC model that proved to be very successful in graphite. ${ }^{6,12,24}$ The hopping terms considered are shown in the inset of Fig. 1(b). The following values of all band parameters except $\gamma_{1}$ and $\Delta$, which were determined above, were taken from Ref. 24: $\gamma_{0}$ $=3.12 \mathrm{eV}, \gamma_{1}=0.378 \mathrm{eV}, \gamma_{3}=0.29 \mathrm{eV}, \gamma_{4}=0.12 \mathrm{eV}$, and $\Delta=0.015 \mathrm{eV}$. Note that they agree well with the values determined in Ref. 25 using Raman spectroscopy. As it was shown in Refs. 20 and 21 the parameters $\gamma_{3}$ and $\gamma_{4}$ affect the gate voltage dependence of the central frequency of the position and the width of the main peak. In this paper we do not attempt to determine these terms from optical spectra. The doped charge and the Fermi energy can be directly determined for any given gate voltage using the known capacitance of the $\mathrm{SiO}_{2}$ layer. ${ }^{17}$ The standard Kubo formula was used to calculate optical conductance

$$
\begin{aligned}
\operatorname{Re} G(\omega)= & \frac{e^{2} d}{4 \pi^{2}} \sum_{i, j \neq i} \int d \vec{k}\left|v_{x, i j}(\vec{k})\right|^{2} \\
& \times \frac{f\left(\epsilon_{\vec{k}, i}\right)-f\left(\epsilon_{\vec{k}, j}\right)}{\epsilon_{\vec{k}, j}-\epsilon_{\vec{k}, i}} \delta\left(\omega-\frac{\epsilon_{\vec{k}, j}-\epsilon_{\vec{k}, i}}{\hbar}\right)
\end{aligned}
$$

that was eventually Gaussian broadened by $0.02 \mathrm{eV}$, in order 
to match the observed line widths. Here $\epsilon_{\vec{k}, i}(i=1, \ldots, 4)$ are the electronic bands, $v_{x, i j}(\vec{k})$ is the matrix element of the in-plane velocity operator, and $f(\epsilon)=\{\exp [(\epsilon-\mu) / T]+1\}^{-1}$ is the Fermi-Dirac distribution. The chemical potential $\mu$ is determined by the doping level. In the calculations we assumed $T=10 \mathrm{~K}$. The reflectivity spectra were computed based on Fresnel equations using the known optical properties of the $\mathrm{SiO}_{2} / \mathrm{Si}$ substrate.

We begin with a calculation which assumes that the only effect of applying gate voltage is to shift the chemical potential and does not include the gate-induced band gap. In panels (a) and (c) of Fig. 3, the color plots of experimental and calculated spectra of $\Delta R\left(\omega, V_{g}\right)$ are represented. One can notice a quite good correspondence between the energy and the gate voltage dependence of the strong spectral features. Having found that such an agreement is present in the raw reflectivity data, we proceed with a detailed experiment-theory comparison in terms of the optical conductance [Figs. 3(b) and $3(\mathrm{~d})]$. In view of the mentioned possibility that the extracted conductance curves contain a spectrally featureless background, here we subtract from all spectra the same, i.e., gate-voltage-independent smooth curve shown as a dashed line in Fig. 2(b). This curve is chosen in such a way that the corrected $\operatorname{Re} G\left(\omega, V_{g}=100 \mathrm{~V}\right)$ coincides with the theoretical values in the regions around $0.2 \mathrm{eV}$ and $0.6 \mathrm{eV}$, where no sharp structures are expected.

The assignment of the optical conductance structures to interband transitions is given in Fig. 3(d). Apart from the discussed strong peak structures $\mathrm{B}$ and $\mathrm{C}$ there is an onsetlike structure A which corresponds to a transition between the low-energy bands 2 and 3, which has the same origin as the onsetlike structure observed in monolayer graphene. ${ }^{5}$ The onset frequency is twice the Fermi level with respect to the Dirac energy, which is in bilayer graphene proportional to $\left|V_{g}-V_{g 0}\right|$ with a coefficient determined by $\gamma_{0}$. In the measured spectra [Fig. 3(b)] we observe such a structure showing the same (within the experimental uncertainty) dependence on the gate voltage. This confirms that $\gamma_{0}$ is close to the value used in the calculation $(3.12 \mathrm{eV})$. This observation is in accordance with a recent measurement of Li et al. ${ }^{20,21}$ Interestingly, in addition to this we see a second onset-like structure, with the onset energy showing a similar V-shape dependence on the gate voltage but shifted with respect to the structure A by about $\gamma_{1}$. The structure is due to the onset of transition $\mathrm{D}(1 \rightarrow 3)$ for the electron doping and transition $\mathrm{E}$ $(2 \rightarrow 4)$ for the hole doping. There is a significant enhancement of $\operatorname{Re} G(\omega)$ close to the "vertex" point $\omega \approx \gamma_{1}, V$ $\approx V_{g 0}$ where the two onsets are close to each other. ${ }^{7,8}$ One can clearly see a similar structure on the experimental graph. Thus the tight-binding model reproduces most of the features of experimental spectra.

\section{GATE-INDUCED BANDGAP: EXPERIMENT VERSUS CALCULATIONS}

Now we address the issue of the gate-induced band gap $\Delta_{g}$ between the low-energy electron and hole bands. ${ }^{13-15}$ Its manifestation in the infrared spectra was first calculated (assuming that $\gamma_{3}, \gamma_{4}$, and $\Delta=0$ ) in Ref. 9. In Fig. 3(f) we show the result of a calculation where we keep the all aforementioned band parameters and add a gate-dependent difference in electrostatic potential between the two planes. We use a curve $\Delta_{g}\left(V_{g}\right)$ from Ref. 17, shown as a red line in Fig. 3(f), where the charge screening effects were treated selfconsistently. We assume that, as it was also done in Ref. 17, contaminant molecules shifting the charge neutrality point away from $V_{g}=0$ act as an effective top-gate electrode. In this case the band gap vanishes not at $V_{g}=V_{g 0}$ but at $V_{g}$ $=-V_{g 0}$. At the highest gate voltages of our experiment the gap value is expected to be on the order of $0.1 \mathrm{eV}$.

According to the calculation, the opening of the band gap indeed brings some extra features to the spectra. All of them are due to the flattening of bands 2 and 3, as shown in Fig. $3(\mathrm{e})$, which results in a strong increase in the density of states of these bands. The first feature (marked $\mathrm{A}^{\prime}$ ) is an enhancement of the optical intensity of the transition $2 \rightarrow 3$. Although this enhancement largely shows up at photon energies below the experimentally accessible region, its tail spreads up to about $0.2 \mathrm{eV}$. The second feature is the appearance of highfrequency satellites (marked $\mathrm{E}^{\prime}$ and $\mathrm{D}^{\prime}$ ) to the peaklike structures $\mathrm{B}$ and $\mathrm{C}$. These satellites correspond to transitions $2 \rightarrow 4$ and $1 \rightarrow 3$, respectively. The energy separation between the central frequencies of peaks $B$ and $E^{\prime}$ as well as between $\mathrm{C}$ and $\mathrm{D}^{\prime}$ is close to the energy of the band gap and could be therefore read directly from the conductance curves. Note that the interband structures $\mathrm{A}^{\prime}, \mathrm{E}^{\prime}$, and $\mathrm{D}^{\prime}$ involve the same band pairs as the structures A, E, and D, respectively. However the former ones are exclusively due to transitions within a very small momentum region around the Dirac point.

We notice that experimental spectra [Fig. 3(b)] show an enhancement of conductance similar to the high-frequency tail of the structure $A^{\prime}$. However the satellite structures $E^{\prime}$ and $\mathrm{D}^{\prime}$ are not obviously present in the data.

\section{DISCUSSION AND OUTLOOK}

Based on Secs. I-V, we state that the tight-binding model is quite successful in describing the main infrared features, but it is only in partial agreement with the data as far as the band-gap-related features are concerned. This fact is perhaps the largest surprise of our study. We can only speculate about the possible reasons. First of all, the satellite features might be smeared out by doping inhomogeneity due to the flake corrugation, contaminant molecules, or other factors. However, the calculation already takes a large broadening (about $0.02 \mathrm{eV}$ ) into account. A second possibility is that the actual band gap is smaller than the prediction of a simple model that does not take into account interaction effects, so that the satellites $\mathrm{E}^{\prime}$ and $\mathrm{D}^{\prime}$ cannot be easily separated from the main peaks. A third possibility is that the gap can be partially filled with impurity states. ${ }^{26}$ Finally, we assumed that the temperature of the graphene flake is the same as the one of the substrate $(10 \mathrm{~K})$. However, graphene can be somewhat warmer, which would also affect optical conductance. Future experimental and theoretical developments are certainly required to finally resolve the intriguing issue of the gatetunable band gap in bilayer graphene. 


\section{ACKNOWLEDGMENTS}

This work was supported by the Swiss National Science Foundation through the National Center of Competence in
Research "Materials with Novel Electronic PropertiesMaNEP." We are grateful to A. Morpurgo, L. Benfatto, E. Cappelluti, and M. Fogler for helpful discussions.
${ }^{1}$ K. S. Novoselov, A. K. Geim, S. V. Morozov, D. Jiang, Y. Zhang, S. V. Dubonos, I. V. Grigorieva, and A. A. Firsov, Science 306, 666 (2004).

${ }^{2}$ A. K. Geim and K. S. Novoselov, Nature Mater. 6, 183 (2007).

${ }^{3}$ T. Ando, Y. Zheng, and H. Suzuura, J. Phys. Soc. Jpn. 71, 1318 (2002).

${ }^{4}$ R. R. Nair, P. Blake, A. N. Grigorenko, K. S. Novoselov, T. J. Booth, T. Stauber, N. M. R. Peres, and A. K. Geim, Science 320, 1308 (2008).

${ }^{5}$ Z. Q. Li, E. A. Henriksen, Z. Jiang, Z. Hao, M. C. Martin, P. Kim, H. L. Stormer, and D. N. Basov, Nat. Phys. 4, 532 (2008).

${ }^{6}$ A. B. Kuzmenko, E. van Heumen, F. Carbone, and D. van der Marel, Phys. Rev. Lett. 100, 117401 (2008).

${ }^{7}$ J. Nilsson, A. H. Castro Neto, F. Guinea, and N. M. R. Peres, Phys. Rev. Lett. 97, 266801 (2006).

${ }^{8}$ D. S. L. Abergel and V. I. Fal'ko, Phys. Rev. B 75, 155430 (2007).

${ }^{9}$ E. J. Nicol and J. P. Carbotte, Phys. Rev. B 77, 155409 (2008).

${ }^{10}$ Z. Jiang, E. A. Henriksen, L. C. Tung, Y.-J. Wang, M. E. Schwartz, M. Y. Han, P. Kim, and H. L. Stormer, Phys. Rev. Lett. 98, 197403 (2007).

${ }^{11}$ F. Wang, Y. Zhang, C. Tian, C. Girit, A. Zettl, M. Crommie, and Y. R. Shen, Science 320, 206 (2008).

${ }^{12}$ J. W. McClure, Phys. Rev. 108, 612 (1957); J. C. Slonczewski and P. R. Weiss, ibid. 109, 272 (1958).

${ }^{13}$ E. McCann and V. I. Falko, Phys. Rev. Lett. 96, 086805 (2006).
${ }^{14}$ F. Guinea, A. H. Castro Neto, and N. M. R. Peres, Phys. Rev. B 73, 245426 (2006).

${ }^{15}$ E. McCann, Phys. Rev. B 74, 161403(R) (2006).

${ }^{16}$ T. Ohta, A. Bostwick, T. Seyller, K. Horn, and E. Rotenberg, Science 313, 951 (2006).

${ }^{17}$ E. V. Castro, K. S. Novoselov, S. V. Morozov, N. M. R. Peres, J. M. B. Lopes dos Santos, J. Nilsson, F. Guinea, A. K. Geim, and A. H. Castro Neto, Phys. Rev. Lett. 99, 216802 (2007).

${ }^{18}$ J. B. Oostinga, H. B. Heersche, X. Liu, A. F. Morpurgo, and L. M. K. Vandersypen, Nature Mater. 7, 151 (2008).

${ }^{19}$ A. B. Kuzmenko, Rev. Sci. Instrum. 76, 083108 (2005).

${ }^{20}$ Z. Q. Li, E. A. Henriksen, Z. Jiang, Z. Hao, M. C. Martin, P. Kim, H. L. Stormer, and D. N. Basov, Phys. Rev. Lett. 102, 037403 (2009)

${ }^{21}$ L. M. Zhang, Z. Q. Li, D. N. Basov, M. M. Fogler, Z. Hao, and M. C. Martin, Phys. Rev. B 78, 235408 (2008).

${ }^{22}$ D. D. L. Chung, J. Mater. Sci. 37, 1475 (2002).

${ }^{23}$ G. Guizzetti, L. Nosenzo, E. Reguzzoni, and G. Samoggia, Phys. Rev. Lett. 31, 154 (1973).

${ }^{24}$ B. Partoens and F. M. Peeters, Phys. Rev. B 74, 075404 (2006).

${ }^{25}$ L. M. Malard, J. Nilsson, D. C. Elias, J. C. Brant, F. Plentz, E. S. Alves, A. H. Castro Neto, and M. A. Pimenta, Phys. Rev. B 76, 201401(R) (2007).

${ }^{26}$ J. Nilsson and A. H. Castro Neto, Phys. Rev. Lett. 98, 126801 (2007). 